\title{
NUEVOS DATOS SOBRE CANTHON (COLEOPTERA: SCARABAEINAE) DE CHIAPAS, MÉXICO
}

\author{
Violeta HALFFTER y Gonzalo HALFFTER \\ Instituto de Ecología, A.C., Km. 2.5 Carretera Antigua a Coatepec No. 351, Congregación El Haya, \\ 91070 Xalapa, Veracruz, MÉXICO. \\ Autor para correspondencia: gonzalo.halffter@inecol.edu.mx
}

Halffter, V., y G. Halffter. 2009. Nuevos datos sobre Canthon (Coleoptera: Scarabaeinae) de Chiapas, México. Acta Zoológica Mexicana (n. s.), 25(2): 397-407.

RESUMEN. Dentro del género Canthon se describe una nueva especie (C. lucreciae), se señala por primera vez la presencia en Chiapas de una segunda especie y se confirma la de dos más conocidas hasta la fecha por un solo registro.

Palabras clave: Canthon, Scarabaeinae, Chiapas.

Halffter, V., \& G. Halffter. 2009. New data for Canthon (Coleoptera: Scarabaeinae) from Chiapas, Mexico. Acta Zoológica Mexicana (n. s.), 25(2): 397-407.

ABSTRACT. For the genus Canthon, a new species (C. lucreciae) is described; for the first time the presence of a second species in Chiapas, Mexico is indicated; as is the presence of two more species previously only known as one record.

Key words: Canthon, Scarabaeinae, Chiapas.

\section{INTRODUCCIÓN}

Antes de este artículo se conocían de Chiapas 17 especies y subespecies de Canthon y 31 de Canthonini (Coleoptera: Scarabaeinae) en conjunto (Thomas, 1993; Halffter, 2003; Chamé-Vázquez y Gómez, 2005; Navarrete y Halffter, 2008a, 2008b). La Selva Lacandona, situada hacia la frontera con Guatemala, ha sido especialmente colectada por varios autores y en diferentes tiempos (en el sur de la Selva Lacandona se ha encontrado la mayor diversidad puntual de Scarabaeinae del país. Navarrete y Halffter, 2008b). Por el contrario, hay pocos esfuerzos de captura sistemática en las formaciones de bosques deciduos o subdeciduos de la Depresión Central de Chiapas, así como en las extensas y muy variadas montañas del estado y en las selvas siempreverdes que se extienden hacia los límites con los estados de Oaxaca y Veracruz. Así, en la extensa región de Los Chimalapas nunca se ha realizado una recolección sistemática de Scarabaeinae.

En este trabajo presentamos las novedades taxonómicas y de distribución derivadas de tres programas de captura intensiva, el realizado por la Dra. Lucrecia

Recibido: 6/11/2008; aceptado: 27/04/2009. 
Arellano (Instituto de Ecología, A.C.) en bosques deciduos y subdeciduos y formaciones antropizadas derivadas de la Depresión Central de Chiapas; y los realizados en dos ocasiones por grupos encabezados por Gonzalo Halffter en bosque perennifolio en Palenque, noroeste del estado de Chiapas. Incluimos únicamente aquellas especies que antes no habían sido señaladas para Chiapas o nuevas para la ciencia, o bien publicadas recientemente de una sola localidad.

\section{MATERIAL Y MÉTODOS}

Se examinaron aproximadamente 2000 ejemplares de Canthon procedentes de la Depresión Central de Chiapas y de la parte norte de la Selva Lacandona (Palenque). Todo este material está depositado en las colecciones de El Colegio de la Frontera Sur (San Cristóbal las Casas, México), Instituto de Ecología, A.C. (Xalapa, México) y Halffter (Xalapa, México). Del material examinado 159 ejemplares corresponden a $C$. lucreciae y 169 a las otras especies estudiadas.

Las microfotografías fueron tomadas con microscopio electrónico de barrido (Jeol JMS-5600 LV) en el Instituto de Ecología, A.C. (Tec. Tiburcio Laez Aponte). Las piezas fueron pasadas por acetona y gas carbónico antes de ser metalizadas con oro.

\section{RESULTADOS Y DISCUSIÓN}

Canthon (Glaphyrocanthon) delgadoi Rivera-Cervantes y Halffter, 1999. Esta especie fue descrita de la costa del estado de Oaxaca (Pochutla, Puerto Ángel) y capturada en bosque tropical caducifolio perturbado (Rivera-Cervantes y Halffter 1999). Se cita por primera vez para Chiapas donde ha sido colectada en varias localidades y en número importante.

Los ejemplares de Chiapas presentan amplia variación en ciertos caracteres y algunas diferencias con el material tipo de Oaxaca. En conjunto los ejemplares de Chiapas son algo mayores que la serie típica: $5.66 \mathrm{~mm}$ de longitud total promedio (entre 5 y $6.5 \mathrm{~mm}$ ), contra una longitud máxima de $5 \mathrm{~mm}$ en la serie típica. En conjunto en el material de Chiapas la superficie dorsal es menos brillante y con el chagrinado más definido y la coloración más obscura (entre verde obscuro y verde negruzco, casi negra o azul en algunos ejemplares) que el material de la serie típica. Sin embargo, entre los numerosos ejemplares de Chiapas se encuentran individuos claramente verdes, color dominante en Oaxaca.

En el borde anterior del clípeo en algunos de los ejemplares de Chiapas se presenta una angulosidad a cada lado de los dientes centrales (Fig. 1). Esta angulosidad forma una indicación (no bien definida) de un segundo par de dientes. Esta angulosidad no se encontró en ninguno de los ejemplares de Oaxaca. En los ejemplares de Chiapas la línea supraclipeal que se extiende inmediatamente detrás de los dientes clipeales, puede ser completa o interrumpida por la muesca que separa los 


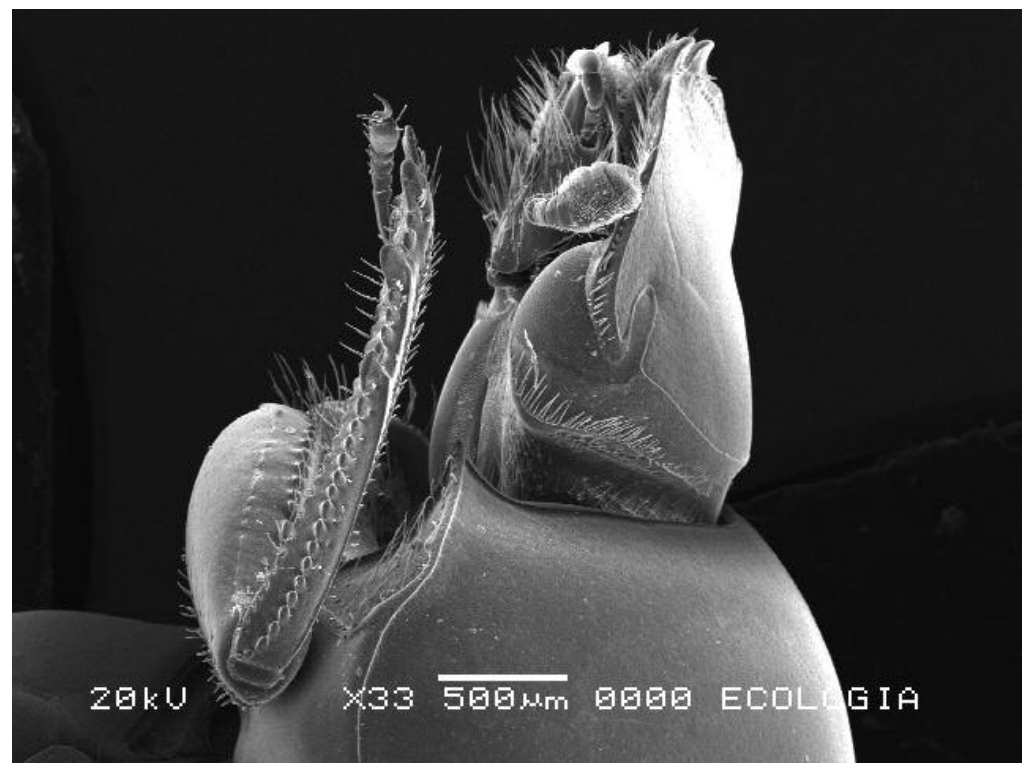

Figura 1. Canthon (Glaphyrocanthon) delgadoi Rivera-Cervantes y Halffter. Vista lateral de la cabeza, nótese la angulosidad detrás de los dientes clipeales y la línea supraclipeal interrumpida.

dientes clipeales. En la serie típica es incompleta y más corta. En algún ejemplar de Chiapas la maza antenal es negra; en la serie típica es de color castaño-rojizo. En algún ejemplar de Chiapas la quilla del borde anterior del fémur I no presenta denticulación completa; en la serie típica es completa. Asimismo, en algún ejemplar de Chiapas la quilla ventral del fémur I es completa; en la serie típica es incompleta.

Pese a las diferencias antes señaladas que son evidencia de una mayor variación en el material de Chiapas, consideramos que éste es claramente coespecífico con la serie típica de $C$. (Gl.) delgadoi procedente de Oaxaca.

Material examinado. MÉXICO: Chiapas: Vicente Guerrero, Municipio San Fernando, 919 m alt., 52 ejemplares de varias fechas entre mayo y septiembre 2004, 2005; La Chacona, Municipio San Fernando, 927 m alt., 16-18/IX/2004, L. Arellano y M. Girón col., 6 ejs.; Chicoasén, 842 m alt., 1/V/2004, L. Arellano y K. Leal col., 1 ej.; 16/IX, 21-25/VII/2003, L. Arellano y A. Molina col., 41 ejs. Todo el material se encuentra en las colecciones del Colegio de la Frontera Sur, San Cristóbal de Las Casas, Chiapas, y Halffter, Xalapa, Veracruz.

Observaciones ecológicas. El material de Vicente Guerrero procede de potreros con o sin árboles aislados, cercos vivos y acahuales de selva baja caducifolia. El material de 16 de Septiembre (Rancho San Pedro) procede de formaciones maduras de Acacia pennatula. Los ejemplares de La Chacona y de Chicoasén proceden de 
selva baja caducifolia. En el mismo tipo de vegetación se encontraron los ejemplares de Oaxaca. Como varias otras especies del grupo viridis, $C$. $(G l$.) delgadoi tiene una amplia tolerancia a los cambios en el tipo de vegetación, lo que le permite vivir en la selva baja caducifolia y en las formaciones mucho más insoladas resultado de la intervención humana. La distribución geográfica de $C$. (Gl.) delgadoi sigue la del bosque tropical deciduo o selva baja caducifolia en esa parte de México, pasando del declive al Pacífico en el Istmo de Tehuantepec (Oaxaca) a la Depresión Central de Chiapas.

El material se colectó en trampas de caída cebadas con excremento humano, de vaca y calamares en descomposición, sin diferencias notables entre los distintos cebos. Una vez más $C$. (Gl.) delgadoi muestra una amplia tolerancia en sus requerimientos ecológicos.

Canthon (Canthon) lucreciae Halffter y Halffter, sp. nov.

Descripción. Holotipo macho: Longitud total $5 \mathrm{~mm}$. Cuerpo oval fuertemente globular (Fig. 2). Coloración de la superficie dorsal verde, francamente brillante en el pronoto. Superficie ventral del tórax verde obscuro, más obscuro hacia los lados, con reflejos brillantes en la zona media del metasternón; último esternito abdominal y pigidio verdes. Patas café negruzcas, con brillos verdes marcados en los fémures de los tres pares de patas; tarsos cafés. Maza de las antenas de color anaranjado.

Cabeza. Borde anterior bidentado, los dientes poco agudos separados por una muesca en V; la línea supraclipeal cubre la base de los dientes. Suturas clípeo-genales bien definidas pero poco profundas. Mejillas en arco, con un muy pequeño dentículo inmediatamente después de la sutura clípeo-genal. Ojos pequeños, alargados. Borde cefálico posterior marginado por un fino surco. La superficie dorsal de la cabeza chagrinada, con puntuación fina pero claramente visible. Estructura ventral del clípeo en forma de quilla que une la base de los dientes. Borde anterior del submentón escotado en $\mathrm{U}$ poco profunda. Separación entre submentón y gula en forma de $\mathrm{V}$ situada en sentido posterad. Maza de las antenas de color anaranjado.

Tórax. Pronoto transversal, con los ángulos anteriores agudos, pero de base ancha. Bordes laterales angulosos, con el ápice del ángulo situado entre el centro y el borde posterior. Sin impresión antescutelar. Bordes anterior y laterales claramente rebordeados. Borde posterior sin reborde, excepto una muy fina señal hacia la parte media. Ángulos posteriores marcados por un pequeño saliente de forma angular. Superficie del pronoto con chagrinado muy fino y aplanado y puntuación muy fina y dispersa. Hypomeron poco excavado, con una fina quilla transversal. El borde externo con un fino dentículo en su parte media prolongado en un poco marcado engrosamiento hacia el ángulo anterior. Zona media del metasternón con chagrinado muy aplastado y puntos muy finos dispersos. Zonas laterales con el chagrinado más definido. Elitros: Con nueve estrías incluyendo la supraepipleural; la octava sólo 


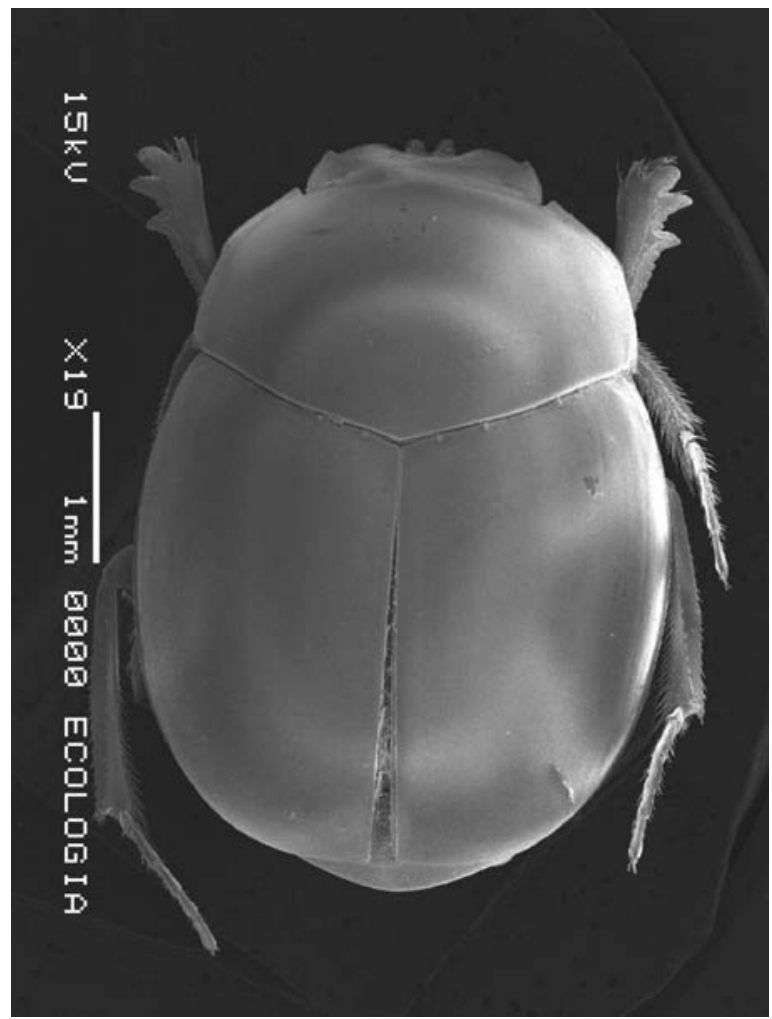

Figura 2. Canthon (Canthon) lucreciae sp. nov. Vista dorsal.

marcada en la base del élitro. Estrías muy finas de color negro. Superficie claramente chagrinada, con puntuación fina apenas visible. Patas: Fémur I con el borde anterior con una quilla entera, sin denticulación (Fig. 3). Superficie ventral chagrinada, con algunos puntos setígeros hacia la base. Tibia I gradualmente ensanchada hacia el ápice, éste truncado en forma oblicua; borde externo con tres dientes, los dos anteriores más aproximados entre sí que el medio y el basal; espolón tibial anterior alargado y delgado, bifurcado en el ápice; tarsos gráciles. Fémur II con una marginación poco marcada en el borde anterior. Superficie con chagrinado muy poco marcado, con muy pocos puntos dispersos. Fémur III con marginación en el borde anterior que se diluye hacia el ápice (Fig. 4). Superficie como en el fémur II. Primer segmento tarsal menor al segundo. Uñas pequeñas rectas.

Abdomen. Esternitos con chagrinado poco marcado, el ultimo más liso y con puntos dispersos. Pigidio con una quilla que margina la base (Fig. 5), chagrinado y con puntuación dispersa. Edeago según Figura 6. 
Halffter y Halffter: Canthon nuevos para Chiapas

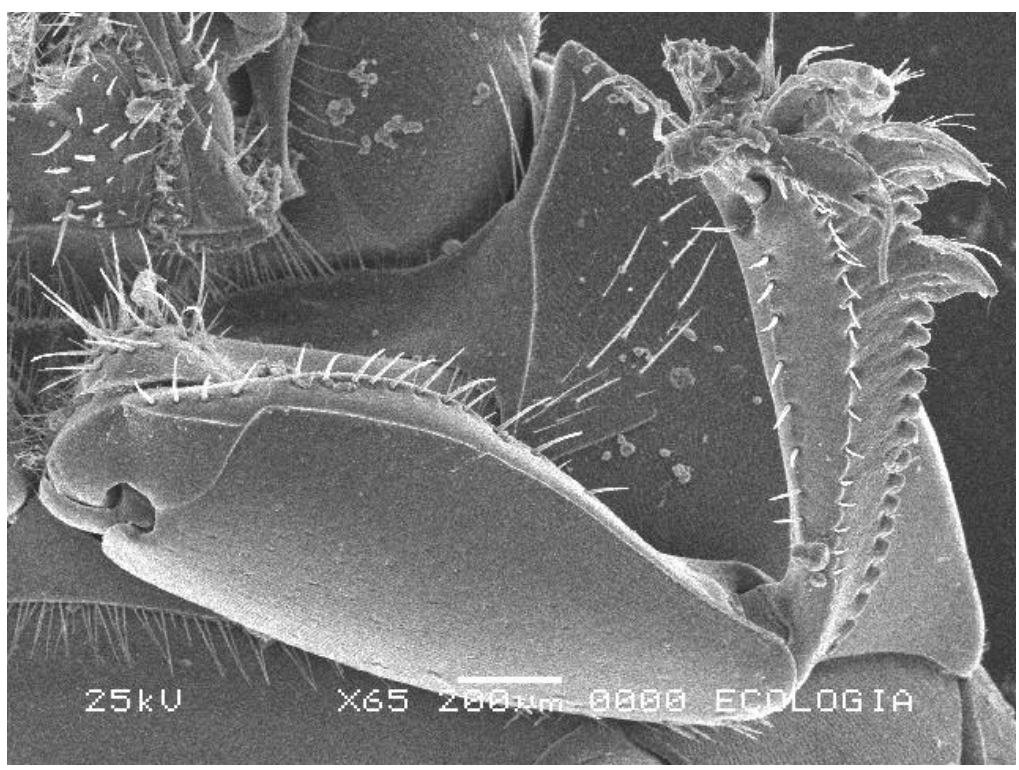

Figura 3. Canthon (Canthon) lucreciae sp. nov. Vista ventral de la pata anterior mostrando la quilla del borde anterior del fémur.

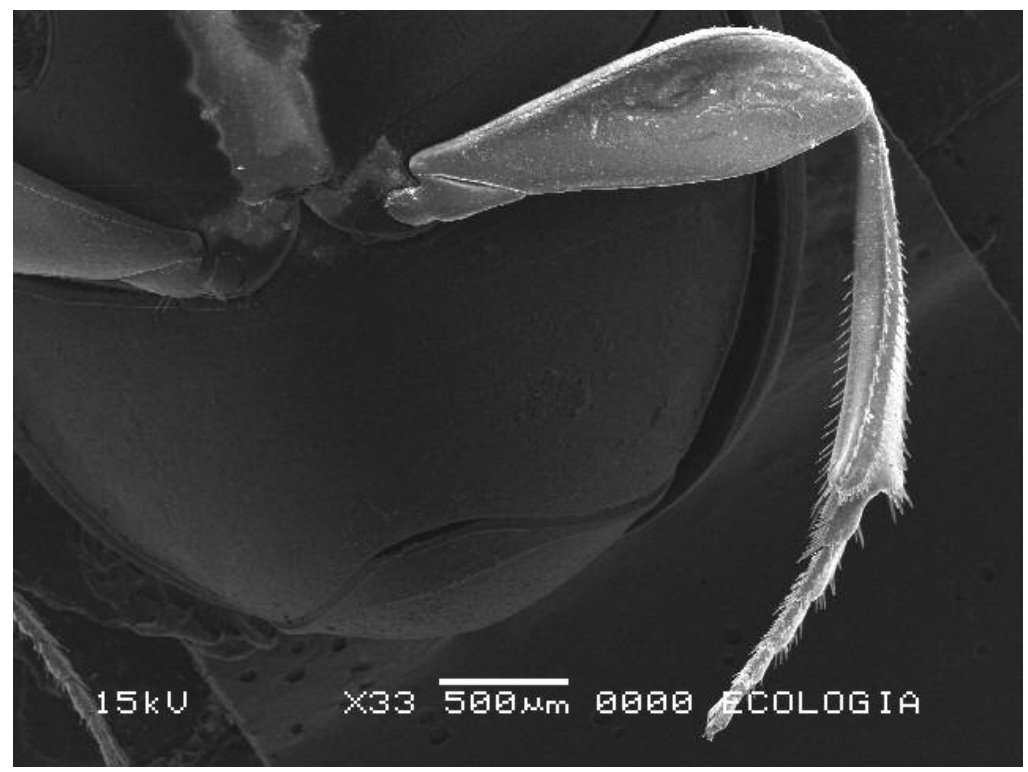

Figura 4. Canthon (Canthon) lucreciae sp. nov. Vista ventral del metafémur mostrando la marginación del borde anterior. 


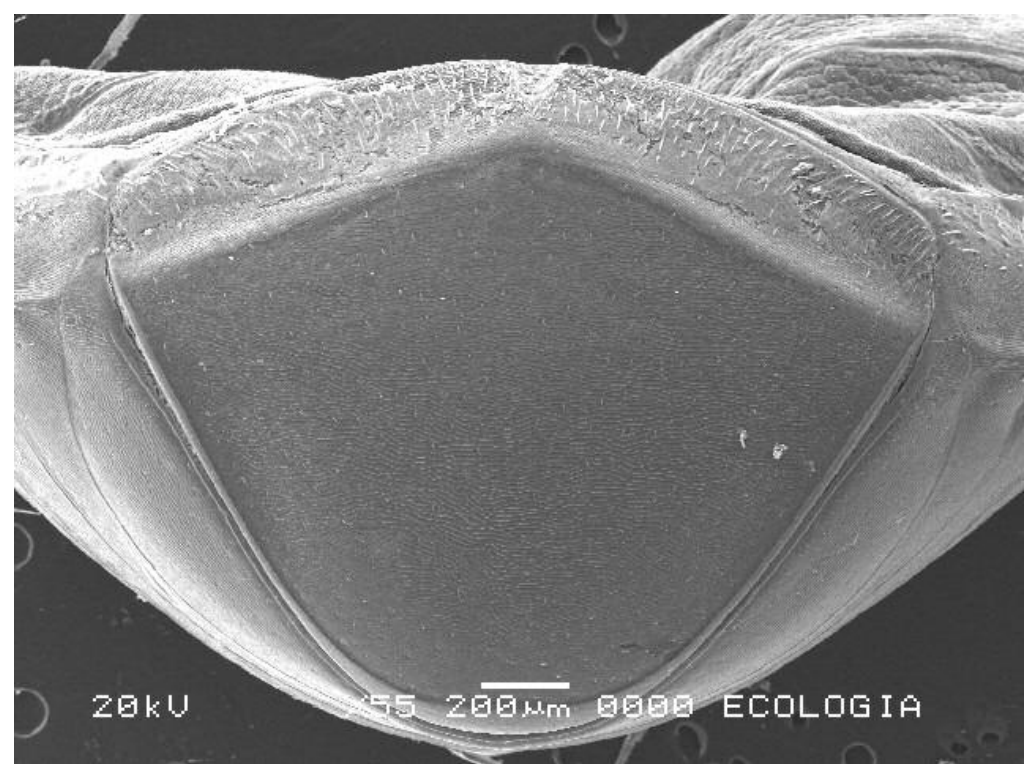

Figura 5. Canthon (Canthon) lucreciae sp. nov. Separación entre pigidio y prepigidio.

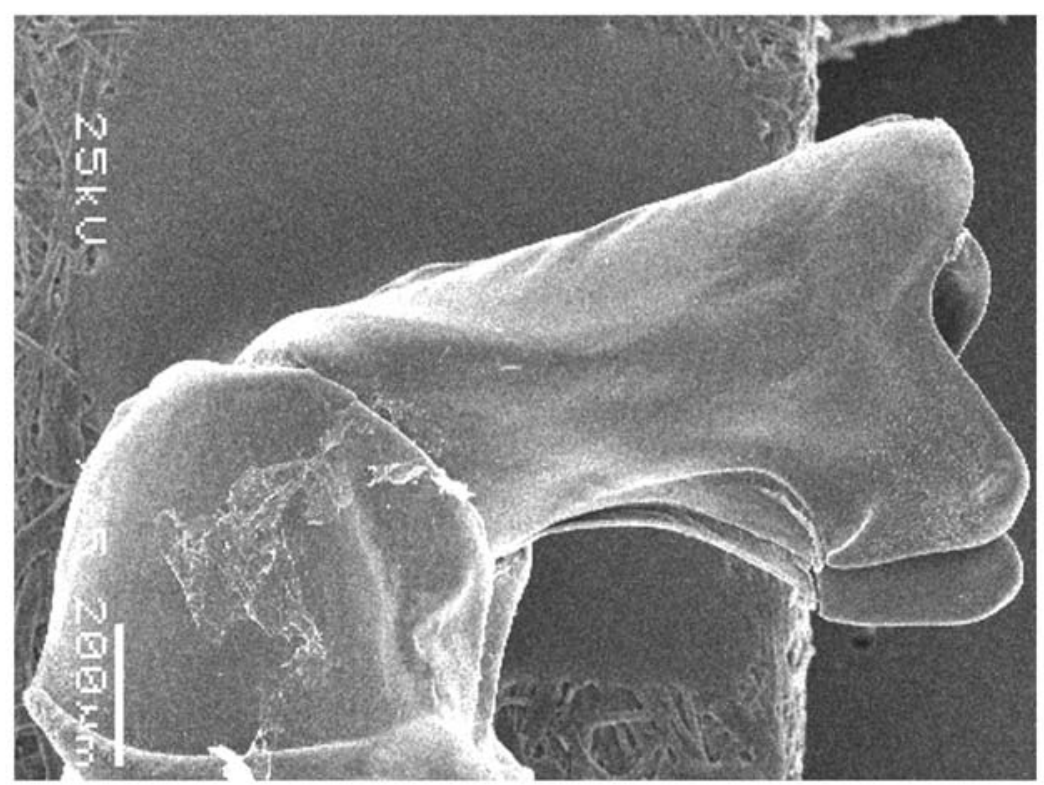

Figura 6. Canthon (Canthon) lucreciae sp. nov. Vista ápico-lateral del edeago. 
Alotipo hembra. Difiere del macho en el espolón de las tibias anteriores que es agudo en el ápice. Además el último esternito abdominal es ancho, sin estrechamiento en la parte media como ocurre en el macho.

Variación. La longitud total varía entre 5 y $6.5 \mathrm{~mm}$. En algunos ejemplares la cabeza y el pronoto presentan brillos dorados o cobrizos; las patas pueden ser verdes y los esternitos abdominales de color verde obscuro. También en algunos ejemplares los dientes clipeales pueden ser más agudos que lo señalado; el diente de las mejillas estar claramente señalado; la puntuación del pronoto estar bastante bien definida y el espolón de la tibia anterior del macho ser espatulado, levemente bifurcado en el ápice. La línea que margina el borde anterior de la cara ventral del fémur posterior, aunque siempre presente, puede ser muy fina y muy próxima al propio borde, lo que dificulta su visión. Esta línea está más definida en la base y se convierte en más difícil de apreciar hacia el ápice.

Etimología. Dedicamos esta especie a la Dra. Lucrecia Arellano (Instituto de Ecología, A.C.), quien ha realizado un profundo trabajo de colecta con interesantes resultados ecológicos y faunísticos en la Depresión Central de Chiapas.

Material examinado. Holotipo: CHIAPAS, Municipio San Fernando, frente a planta de luz, 23-IX-2003, J. León Cortés y M. Girón col. Alotipo: CHIAPAS, Municipio San Fernando, Vicente Guerrero, La Herradura, 18-IX-2004, L. Arellano y K. Leal col. 116 paratipos de CHIAPAS: Municipio San Fernando, La Aguaná, 23IX-2003, J. León Cortes y M. Girón col., 1 macho; Municipio San Fernando, Vicente Guerrero, varias colectas entre junio y septiembre 2004, 2005; Municipio San Fernando, La Chacona, 18-IX-2004, L. Arellano y M. Girón col.

Holotipo y Alotipo en la colección Halffter (Xalapa). Paratipos en la misma colección y en las colecciones del Colegio de la Frontera Sur, San Cristóbal Las Casas, Chiapas, Morón (Xalapa) y Vaz de Mello (Cuiaba, Brasil).

Hasta ahora $C$. lucreciae se ha colectado en formaciones secundarias (acahuales) de bosque tropical deciduo, dentro del mosaico de tipos de vegetación con profunda influencia antrópica que es la Depresión Central de Chiapas, entre 600 y 900 m altitud. En cuanto a la alimentación, según los datos de las trampas de captura parece ser una especie generalista.

Afinidades. Especie muy similar a Canthon indigaceaus chevrolati Harold con la que coincide en la facies y en muchos caracteres de importancia taxonómica. Difiere en la longitud total que es marcadamente menor, incluso a la de los ejemplares de $C$. i. chevrolati más pequeños de Mapastepec, Chiapas (véase Halffter 1961); difiere además por la presencia de quilla, aun abreviada, en el hypomeron; por presentar un tubérculo en el borde externo del mismo; por tener los bordes laterales del pronoto claramente angulosos; y por el chagrinado de la superficie del élitro.

Canthon lucreciae presenta una convergencia en su facies general con Canthon (Glaphyrocanthon) euryscelis Bates, del que C. lucreciae difiere por presentar una 
fina línea marginando el borde anterior (vista ventral) del metafémur, carácter que lo sitúa en el subgénero Canthon. Además de pequeñas diferencias en la coloración de la superficie dorsal del cuerpo (en $C$. (Gl.) euryscelis en general más obscura), la forma de los edeagos es distinta.

De Canthon morsei Howden, otra especie de pequeño tamaño de los trópicos mexicanos, se distingue $C$. lucreciae por tener el pigidio separado del prepigidio por una quilla algo angular en su parte central, quilla que falta o es muy poco conspicua en $C$. morsei. Sin embargo, hay que señalar que lo que se viene considerando en la literatura como $C$. morsei no es una especie, sino un conjunto de especies con variación de ciertos caracteres como la quilla antes referida.

\section{Canthon (Canthon) morsei Howden, 1966.}

Se confirma la presencia dentro del estado de Chiapas de esta especie. Hemos examinado el siguiente material: CHIAPAS: Palenque, 17 junio 1993, G. Halffter et al. col., 1 ejemplar capturado en borde bosque perennifolio, en trampa diurna (13-18 hrs) con excremento; idem, 18 junio 1993, G. Halffter et al. col., 1 ejemplar capturado en bosque perennifolio, en trampa nocturna con excremento; idem, 1 ejemplar en trampa nocturna con carroña; idem, 1 ejemplar capturado en borde bosque perennifolio, en trampa diurna con pescado en descomposición.

Palenque se encuentra en el extremo noroeste de la gran extensión de selva perennifolia conocida como Selva Lacandona. Muy recientemente, Navarrete y Halffter (2008b) citan esta especie del extremo sur de la misma Selva.

Canthon morsei descrito originalmente de Fortín de las Flores (Veracruz) extiende su área de distribución hasta Panamá (inclusive), asociado a bosques tropicales de distinto tipo. Sin embargo, en esta extensa área es muy posible que se encuentre no una especie sino un conjunto de especies muy próximas, pero que difieren en algunos caracteres importantes como la presencia o no de una fina quilla separando prepigidio de pigidio.

En la descripción original se señala: "Pygidium... separated from propygidium by a small carina" (Howden, 1966). Todos los ejemplares que hemos visto de México (Veracruz y Chiapas) tienen una separación entre prepigidio y pigidio, aunque ésta puede estar sólo señalada por un leve engrosamiento y un muy fino surco. Estos ejemplares corresponderían al C. morsei Howden s. str.

Howden y Young (1981) se refieren a las formas panameñas como "Canthon morsei group (Panama form)", señalando que carecen de quilla basal en el pigidio, "in this respect differing from Mexican morsei". Además de México y Panamá, citan la especie de El Salvador y Ecuador. En 1987, Howden y Gill señalan "Additional material from southern Mexico and Costa Rica indicates that the Panama form of morsei represents only one partition of a gradual north-south cline". En el mismo trabajo describen una segunda especie del mismo complejo (Canthon hartmanni Howden y Gill) de un área de Panamá y partes cercanas de Costa Rica. 
Solís y Kohlmann (2002) señalan C. morsei de varias localidades de Costa Rica, indicando que el pigidio no presenta una carina basal. Halffter (2003) vuelve a señalar la especie de México, incluyendo una referencia en cuadro a su presencia en Chiapas pero sin indicar localidad. Avendaño-Mendoza et al. (2005) señalan $C$. morsei del norte de Guatemala.

Como se ha indicado, las capturas de México corresponden a lo que sería $C$. morsei Howden $s$. str. Está pendiente un análisis detallado de la variación asociada a la distribución geográfica, para determinar que status taxonómico se reconoce para la forma (o formas) centroamericanas y sudamericanas.

Canthon (Glaphyrocanthon) angustatus Harold, 1867.

La primera cita para México (Chiapas) es dada por Chamé-Vázquez y Gómez (2005). Navarrete y Halffter (2008b) citan esta especie de la parte sur de la Selva Lacandona, en la Reserva de la Biosfera Montes Azules.

Hemos visto material de CHIAPAS: Palenque, 21-VI-1993, 31 ejs., Halffter et al. col. interior selva perennifolia, en cebo con excremento humano situado a $5 \mathrm{~m}$ de altura; idem, 18 ejs., en cebo con excremento humano situado a $15 \mathrm{~m}$ de altura; idem, 10 ejs., en cebo con excremento humano situado a $20 \mathrm{~m}$ de altura, idem, 19-VI-1993, 5 ejs., G. Halffter et al. col., en excremento situado en el suelo, colectados entre 8 y 10:30 hrs.

Tanto los ejemplares de Montes Azules (Navarrete y Halffter, 2008b) como los de Palenque, han sido colectados en el interior de selva perennifolia, muy abundantes cuando hay monos. Esta situación se repite en otros lugares de su área de distribución (ver Solís y Kohlmann, 2002). Buena parte de los ejemplares de Palenque volaban en el interior de la selva, donde en su momento se encontraba un grupo de monos. Fueron colectados mediante cebos suspendidos entre los 5 y los $20 \mathrm{~m}$ de altura. En el mismo lugar, el mismo comportamiento "aéreo" se encuentra en Canthon (Glaphyrocanthon) euryscelis Bates, $C$. (Gl.) subhyalinus Harold y $C$. (Gl.) femoralis Chevrolat.

Canthon (Gl.) angustatus tiene una amplia distribución desde Perú y Ecuador hasta Guatemala, Belice y Chiapas (México). Como en el caso de Canthon lituratus (Germar, 1813) (véase Navarrete y Halffter, 2008a) se trata de especies sud-centroamericanas claramente asociadas a los bosques tropicales perennifolios o subperennifolios, cuya presencia en Chiapas corresponde al límite norte de su área de distribución y puede considerarse como una extensión biogeográficamente reciente.

AGRADECIMIENTOS. Agradecemos a Yrma López Guerrero, Tiburcio Laez Aponte y a Magdalena Cruz Rosales (Instituto de Ecología, A.C.) su apoyo para la toma de las microfotografías que ilustran esta comunicación. La Dra. Lucrecia Arellano, también del Instituto de Ecología, A.C., nos facilitó el material de la Depresión Central de Chiapas. Los Dres. Mario E. Favila y J. Lobo tuvieron una participación muy activa en las capturas realizadas en Palenque. 


\section{LITERATURA CITADA}

Avendaño-Mendoza, C., A. Morón-Ríos, B. E. Cano \& J. León-Cortés. 2005. Dung beetle community (Coleoptera: Scarabaeidae: Scarabaeinae) in a tropical landscape at the Laucha Region, Guatemala. Biodiversity and Conservation 14(4): 801-822.

Chamé-Vázquez, E. R. \& B. Gómez-Gómez. 2005 Primer registro de Canthon angustatus Harold 1867 en México (Coleoptera: Scarabaeoidea). Acta Zoológica Mexicana (nueva serie) 21 (3): $159-160$.

Halffter, G. 1961. Monografía de las especies norteamericanas del género Canthon Hoffsg. Ciencia (Mex.) 20 (9-12): 225-320.

Halffter, G. 2003. Tribu Scarabaeini. En M.A. Morón (Ed.). Atlas de los Escarabajos de México. Coleoptera: Lamellicornia. Vol. 2, pp. 31-43. Arganis Editio, Barcelona, España.

Howden, H. F. 1966. Notes on Canthonini of the "Biologia Centrali - Americana" and descriptions of new species (Coleoptera: Scarabaeidae). The Canadian Entomologist, 98 (7): 725-741.

Howden, H. F. \& B. D. Gill. 1987. New species and new records of Panamanian and Costa Rican Scarabaeinae (Coleoptera: Scarabaeidae). The Coleopterist Bulletin, 41 (3): 201-224.

Howden, H. F. \& O. P. Young. 1981. Panamanian Scarabaeinae: Taxonomy, distribution and habits (Coleoptera, Scarabaeidae). Contributions American Entomological Institute, 18 (1): 1-204.

Navarrete, D. \& G. Halffter. 2008a. Nuevos registros de escarabajos copro-necrófagos (Coleoptera: Scarabaeidae) para México y Chiapas. Acta Zoológica Mexicana (nueva serie) 24 (1): 247-250.

Navarrete, D. \& G. Halffter. 2008b. Dung beetle (Coleoptera: Scarabaeidae: Scarabaeinae) diversity in continuous forest, forest fragments and cattle pastures in a landscape of Chiapas, México: the effects of anthropogenic changes. Biodiversity and Conservation 17(12): 2869-2898.

Rivera-Cervantes, L. E. \& G. Halffter. 1999. Monografía de las especies mexicanas de Canthon del subgénero Glaphyrocanthon (Coleoptera: Scarabaeidae: Scarabaeinae). Acta Zoológica Mexicana, 77: 23-150.

Solís, A. \& B. Kohlmann. 2002. El género Canthon (Coleoptera: Scarabaeidae) en Costa Rica. Giornale Italiano de Entomologia, 10: 1-68.

Thomas, D. B. 1993. Scarabaeidae (Coleoptera) of Chiapanecan forests: A faunal survey and chronographic analysis. The Coleopterist Bulletin, 47 (4): 363-408. 